\title{
IMPACTS OF THE DETECTION OF CASSIOPEIA A POINT SOURCE
}

\author{
Hideyuki UMeda, Ken'ichi Nomoto \\ Department of Astronomy and Research Center for the Early Universe, University of Tokyo, Bunkyo-ku, Tokyo 113-0033, Japan \\ e-mail: umeda@astron.s.u-tokyo.ac.jp, nomoto@astron.s.u-tokyo.ac.jp \\ SACHIKO TSURUTA \\ Department of Physics, Montana State University, Bozeman, Montana 59717, USA \\ e-mail: uphst@gemini.oscs.montana.edu \\ Shin Mineshige \\ Department of Astronomy, Kyoto University, Sakyo-ku, Kyoto 606-8502, Japan \\ e-mail: minesige@kusastro.kyoto-u.ac.jp
}

\begin{abstract}
Very recently the Chandra First Light Observation discovered a point-like source in Cassiopeia A (Cas A) supernova remnant. This detection was subsequently confirmed by the analyses of the archival data from both ROSAT and Einstein observations. Here we compare the results from these observations with the scenarios involving both black holes (BH) and neutron stars (NS). If this point source is a BH we offer as a promising model a disk-corona type model with low accretion rate where a soft photon source at $\sim 0.1 \mathrm{keV}$ is Comptonized by higher energy electrons in the corona. If it is a NS the dominant radiation observed by Chandra most likely originates from smaller, hotter regions of the stellar surface, but we argue that it is still worthwhile to compare the cooler component from the rest of the surface with cooling theories. We emphasize that the detection of this point source itself should potentially provide enormous impacts on the theories of supernova explosion, progenitor scenario, compact remnant formation, accretion to compact objects, and neutron star thermal evolution.
\end{abstract}

Subject headings: stars: neutron stars — stars: supernovae: general

\section{INTRODUCTION}

Cassiopeia A (Cas A) is an interesting supernova ( $\mathrm{SN})$ remnant in various aspects. The remnant is very young, about 320 years old. This ring-shaped (e.g. Holt et al. 1994) remnant is associated with jet like structures (Fesen, Becker \& Blair 1987). The observed abundances of heavy elements are in good agreement with the yields of a massive star (e.g., Hughes et al. 2000). The overabundance of nitrogen found in some knots (Fesen et al. 1987) implies that the progenitor was a massive Wolf-Rayet star (WN type) which has lost most of its H-rich envelope during the pre-SN evolution. The SN was suggested to be faint (Ashworth 1980), which implies that the progenitor was not a red-supergiant possibly due to loss of its H-rich envelope.

Recently the ACIS on board the Chandra X-ray satellite observed Cas A and found a point-like source (Tananbaum et al. 1999). Subsequently, Aschenbach (1999) reported that the ROSAT/HRI image of Cas A taken during 19951996 also shows the point-like source at the similar location. Very recently Pavlov et al. (2000) and Chakrabarty et al. (2000) reported the results of their detailed analyses of the Cas A point-source data from the Chandra observation. These authors convincingly argue that the observed point source should, indeed, be a compact remnant of the $\mathrm{SN}$ explosion. The single power-law fit to the Chandra data by Pavlov et al. (2000) yields the higher photon index $\Gamma$ and lower luminosity $L$ than those observed from typical young pulsars. The spectrum can be equally well fit by thermal models. The best fit for a one component blackbody model yields the temperature $T^{\infty}=6-8 \mathrm{MK}$, the effective radius $R_{e}=0.20-0.45 \mathrm{~km}$, and the bolometric luminosity $L^{\infty}=(1.4-1.9) \times 10^{33} \mathrm{erg} \mathrm{s}^{-1}$. (In this paper the temperature $T^{\infty}$ and luminosity $L^{\infty}$ refer to the values to be observed at infinity.) Chakrabarty et al. (2000) obtained similar results. The size is too small for a $10 \mathrm{~km}$ radius neutron star (NS), but it is consistent if the dominant emission comes from localized hot spots. Pavlov et al. (2000) find that the spectrum is equally well fit by a two temperature thermal model with hydrogen polar caps and the rest of the cooling NS surface composed of Fe. These authors also analyzed the archival data from ROSAT and Einstein, and report that the results are consistent with the Chandra results within the $1 \sigma$ level. Their data analyses of the point source showed no statistically significant variability (both long and short time scale) over the Einstein - Chandra period. Chakrabarty et al. (2000) carried out detailed timing analysis and report that the 3 $\sigma$ upper limit on the sinusoidal pulsed fraction is $<25 \%$ for period $P>100 \mathrm{~ms},<35 \%$ for $P>5 \mathrm{~ms}$, and $<50 \%$ for $P>1 \mathrm{~ms}$.

We emphasize here that the detection of the point source itself is extremely important, whether it turns out to be a neutron star (NS) or a black hole (BH). In this paper, therefore, we will consider both cases. Although the currently available data are not sufficient to distinguish between these options, the most recently completed long Chandra observation by S. Holt et al. (2000) and already planned long XMM observations should be able to do so. Therefore, we consider that it is extremely important and timely now, to discuss the implications and offer some predictions for each case.

\section{ACCRETING BLACK HOLE}

If the Cas A progenitor is more massive than $\sim 25 M_{\odot}$ a BH may be formed in the explosion (e.g., Ergma \& van den Heuvel 1998). After formation the inner part of the ejected 
matter may fall back onto the $\mathrm{BH}$ due to the presence of a deep gravitational potential well or a reverse shock. The property of an accreting $\mathrm{BH}$ depends strongly on whether or not an accretion disk is formed. Here we present plausible $\mathrm{BH}$ scenarios based on the disk accretion model under the following observational constraints (see, e.g., Pavlov et al. 2000): (1) the single power-law X-ray luminosity of intermediate brightness, $L_{\mathrm{x}}(0.1-5.0 \mathrm{keV})=(2-60) \times 10^{34}$ $\mathrm{erg} \mathrm{s}^{-1}$ for distance $d=3.4 \mathrm{kpc}$, which is much lower than the Eddington luminosity, $L_{\mathrm{EDD}} \sim 7.5 \times 10^{38}\left(M_{\mathrm{BH}} / 3 M_{\odot}\right)$ $\mathrm{erg} \mathrm{s}^{-1}$ for hydrogen-free matter, (2) no significant variability being detected between the Einstein and Chandra observations, (3) large $F_{\mathrm{x}} / F_{\text {opt }}(\gtrsim 100)$, and (4) large power-law photon index, $\Gamma \sim 2.6-4.1$.

In our model, we assume that the fallback material has specific angular momentum greater than $\sim\left(G M_{\mathrm{BH}} r_{\mathrm{S}}\right)^{1 / 2}$ (where $r_{\mathrm{S}}$ is the Schwarzschild radius) and thus a fallback disk is formed. There is no efficient mechanism for angular-momentum removal since the Cas A compact remnant is unlikely to have a binary companion (§4). Then the disk evolution most likely obeys the self-similar solution in which the total angular momentum within the disk is kept constant (Pringle 1974; Mineshige, Nomoto, \& Shigeyama 1993). This solution predicts that disk luminosity decays in a power-law fashion after the disk is formed (Mineshige et al. 1997) as $l \equiv$ $L / L_{\mathrm{EDD}} \sim 10\left(M_{\text {fallback }} / 0.1 M_{\odot}\right)(\alpha / 0.1)^{-1.3}(t / 320 \mathrm{yr})^{-1.3}$ $\left(M_{\mathrm{BH}} / 3 M_{\odot}\right)^{-1.15}$, where $M_{\text {fallback }}$ is the amount of fallback material and $\alpha$ is the viscosity parameter. We should allow a factor of $0.1-10$ changes depending on the distribution of matter and angular momentum. In order for the black-hole accretion scenario to be consistent with the observed $l \sim 10^{-4}$ at $320 \mathrm{yr}$, the amount of the fallback material should indeed be very small, $M_{\text {fallback }} \sim 10^{-6} M_{\odot}$. Although the accretion models predict luminosity decrease during the last 20 years from the Einstein (in 1979) to the Chandra (in 1999) observations, it is small, only about $10 \%-(320 / 300)^{-1.3} \sim 0.90$. Since the Einstein observations include larger error bars, more than several tens of $\%$, the luminosity drop of this level cannot be detected, which is consistent with the lack of observed long range large scale variability.

The luminosity of $\sim 10^{33} \mathrm{erg} \mathrm{s}^{-1}$ is typical to Galactic $\mathrm{BH}$ candidates $(\mathrm{GBHC})$ during quiescence. However, the constraint (3), the large $F_{\mathrm{x}} / F_{\text {opt }}$ ratio, rules out models that invoke formation of a fallback disk whose properties are similar to those in quiescent GBHC (Chakrabarty et al. 2000). In the case of usual GBHC, hydrogen-rich matter is continuously added to the disk from the binary companion. According to the disk-instability model for outbursts of GBHC (Mineshige \& Wheeler 1989), a part of the transferred material is accumulated in the outer parts of the disk, which inevitably produces large optical flux in the quiescent GBHC. Also the constraint (4), a large photon index $\Gamma$, is in conflict with the ADAF (advectiondominated) model for the quiescent GBHC (Narayan, McClintock, \& Yi 1996). For any ADAF models in which soft photons are provided only by internal synchrotron emission and no external soft photons are available, the powerlaw photon indices should be as small as $\Gamma \sim 1.7$ (Tanaka $\&$ Lewin 1995). These are the reasons why Chakrabarty et al. (2000) did not favor an accreting BH model for the
Cas A source.

Here we propose a different promising $\mathrm{BH}$ model, a diskcorona type model, for which the above analogy to the GBHC is not valid. First, we consider the constraint (3), the large $F_{\mathrm{x}} / F_{\text {opt }}$ ratio. In our model for Cas A, there is no binary companion which supplies mass at 320 years $(\S 4)$. This means that the outer disk boundary is not extended enough to emit significant optical fluxes. The disk is stable due to the smaller disk size and the different composition of the disk material (mostly heavy elements with possibly a little He but no hydrogen; §4), i.e., the thermally unstable outer zones are absent. In the absence of an instability, the mass-flow rate in the disk is close to be constant (Mineshige et al. 1993). Then according to the standard disk model, the effective temperature is $T(r) \sim 4000\left(M_{\mathrm{BH}} / 3 M_{\odot}\right)^{1 / 4}\left(r / 10^{10} \mathrm{~cm}\right)^{-3 / 4}\left(l / 10^{-4}\right)^{1 / 4}$ $\mathrm{K}$. For the disk size as small as $r_{\mathrm{d}} \lesssim 10^{10} \mathrm{~cm}$ and $l \sim 10^{-4}$, the constraint $L_{\mathrm{opt}}<10^{32} \mathrm{erg} \mathrm{s}^{-1}$ is satisfied.

Next consider the constraint (4), the large $\Gamma$. In order to reproduce large photon indices by Compton scattering, we require that energy input rate into soft photons exceeds that into electrons. It is important to note that GBHC generally exhibit two states, soft and hard, and a large $\Gamma$ is a characteristics of the soft-state emission which exhibits soft blackbody spectra with $k T \sim 1 \mathrm{keV}$. The radiation from thermal photons with $\sim 1 \mathrm{keV}$ times the area of the emission region around a typical black hole of $3-10 \mathrm{M}_{\odot}$ produces higher luminosity, $L_{\mathrm{x}} \sim 10^{36-38}$ $\mathrm{erg} \mathrm{s}^{-1}$, than observed from Cas A. However, we emphasize that unlike GBHC no further mass input is available in our model. Then the accretion rate monotonically decreases, and so does the maximum blackbody temperature, as $T_{\max } \sim 0.1\left(M / 3 M_{\odot}\right)^{1 / 4}\left(l / 10^{-4}\right)^{1 / 4} \mathrm{keV}$. Therefore, we get $T_{\max } \sim 0.1 \mathrm{keV}$ for $l \sim 10^{-4}$, instead of $\sim 1 \mathrm{keV}$. A large $\Gamma$ is then naturally obtained in our model, since there is copious supply of soft-photons at $\sim 0.1 \mathrm{keV}$ into electron clouds in the corona from the underlying cool disk (Mineshige, Kusunose, Matsumoto 1995). In other words, the important model parameter is $\ell_{\text {soft }} / \ell_{\text {hard }}$ (ratio of compactness parameter of soft photons to that of hard electrons), where the compactness parameter is proportional to the energy output rate divided by the size of the region. For $\ell_{\text {soft }}>\ell_{\text {hard }}$, we have a large spectral index $(\Gamma>2)$ because of efficient Compton cooling of hard electrons as shown in Mineshige et al. (1995). The spectral slope is rather insensitive to $\dot{M}$ and $M$. The conclusion is that with the low accretion rate and lower soft photon temperature our Compton model with a disk-corona configuration naturally yields large $\Gamma$ with the observed luminosity.

\section{COOLING NEUTRON STAR}

Here let us assume that the observed Cas A point source is a NS. Pavlov et al. (2000) and Chakrabarty et al. (2000) convincingly argue that the dominant radiation observed by Chandra is most likely coming from polar hot spots or equatorial ring if it is a NS. Our main purpose in this section is to argue that it is still worthwhile to compare with theoretical models the observed upper limit to the cooling NS component (i.e., the radiation from the whole stellar surface excluding the hotter, localized areas).

Pavlov et al. (2000) offered, as a possible model, a twocomponent thermal model where the temperature and ra- 
dius of the polar caps with hydrogen are $2.8 \mathrm{MK}$ and $\sim$ $1 \mathrm{~km}$, respectively, while the rest of the surface of the 10 $\mathrm{km} \mathrm{NS}$ consisting of $\mathrm{Fe}$ is at $1.7 \mathrm{MK}$. In this model, the hotter polar caps are the result of higher conductivity of hydrogen as compared with $\mathrm{Fe}$, the temperature difference between the polar caps and the rest of the surface should be small, less than a factor of 2, and hence non-standard cooling should be excluded.

Here we offer a promising alternative NS model. SN remnants are usually classified into two categories: shelltype and filled-center (plerions). Cas A is considered to be a prototype of the former, where radio pulsars are normally not found. Recently Pacini (2000) emphasized the evidence for the presence of an active NS in at least some of the shell-type SNRs although radio pulsars were not found. Also, there is some evidence for significant magnetospheric activities (which can be responsible for polar cap heating) in some NS where no radio pulsar has been found. An example is Geminga (e.g., see Tsuruta 1998). Therefore, the apparent absence of a radio pulsar and/or a plerion should not be used as evidence against polar cap heating. Chakrabarty et al. (2000) offers accretion as a possible cause for polar cap heating when the field strength is significant. If it is weak, their accreting NS model offers the hotter component as originating from the equatorial hot ring. In either case, with an additional heat source for the hotter component, larger temperature difference between the hotter and cooler components is expected, and hence there is no conflict with the possibility of faster nonstandard cooling.

We adopt the conservative upper limit to the cooler component given by Chandara (Pavlov et al. 2000), $L^{\infty}<3 \times 10^{34} \mathrm{erg} \mathrm{s}^{-1}$. The neutron star thermal evolution is calculated with a general relativistic evolutionary code without making the isothermal approximation (Nomoto \& Tsuruta 1987, Umeda et al. 1994, hereafter U94; Umeda, Tsuruta \& Nomoto 1994, hereafter UTN94). Our results are summarized in Figure 1.

The observed upper limit for Cas A is consistent with the 'standard' cooling. However, it is still only an upper limit, and if the actual luminosity of the cooler component turns out to be $\sim 10^{33} \mathrm{erg} \mathrm{s}^{-1}$ or less, the result will be extremely interesting. This is because then the observed value will be certainly below the standard cooling curve, and hence that will be considered the evidence for non-standard cooling scenarios such as those involving pion and/or kaon condensates, or the direct URCA process (e.g., U94; UTN94). When the particles in the stellar core are in the superfluid state with substantial superfluid energy gaps, neutrino emissivity $l_{\nu}$ is significantly suppressed (e.g., see Tsuruta 1998). In order to examine this effect of superfluidity, we calculated pion cooling for a representative superfluid model with an intermediate degree of suppression, called the E1 - 0.6 model (see U94). The result is shown as the thin solid curve in Figure 1.

\section{CONSTRAINTS FROM PROGENITOR SCENARIOS}

Here we discuss whether the formation of a NS or BH is consistent with the current models of stellar evolution and supernovae and whether the evolutionary scenarios constrain the radiation processes from the compact source. The overabundance of nitrogen in Cas A implies that the progenitor was a massive WN star which lost most of its hydrogen envelope before the SN explosion. Here we describe two possible evolutionary paths to form such a preSN WN star.

One path is the mass loss of a very massive single star. A star with the zero-age main-sequence mass $M_{\mathrm{MS}}$ larger than $\sim 40 \mathrm{M}_{\odot}$ can lose its hydrogen-rich envelope via mass loss due to strong winds and become a Wolf-Rayet star (e.g., Schaller et al. 1992). Recent theoretical models and population synthesis studies suggest that stars with $M_{\mathrm{MS}} \gtrsim 25 \mathrm{M}_{\odot}$ are more likely to form BHs than NSs (e.g., Ergma \& van den Heuvel 1998). This implies that the WN star progenitor is massive enough to form a $\mathrm{BH}$. The explosion can be energetic enough to prevent too much matter fall back to be consistent with the small fall back mass inferred in $\S 2$.

The other evolutionary path to form a pre-SN WN star is mass loss due to binary interaction. If the progenitor is in a close binary system with a less massive companion star, the star loses most of its H-rich envelope through Roche lobe overflow. In this case, the WN progenitor can form from a star of $M_{\mathrm{MS}} \lesssim 40 \mathrm{M}_{\odot}$. Its $\mathrm{SN}$ explosion of type $\mathrm{Ib} / \mathrm{c}$ would leave either a $\mathrm{BH}$ (if $M_{\mathrm{MS}} \sim 25-40 \mathrm{M}_{\odot}$ ) or a NS (if $M_{\mathrm{MS}} \lesssim 25 \mathrm{M}_{\odot}$ ). If the compact remnant in Cas A turns out to be a NS, therefore, the progenitor must have been in a close binary system.

In the binary scenario, the companion to the Cas A progenitor cannot be more massive than a red dwarf, as constrained from the R \& I band magnitude limit (van den Bergh \& Pritchet 1986). When the companion star is such a small mass star, i.e., the mass ratio between the stars is large, the mass transfer is inevitably non-conservative (e.g., Nomoto, Iwamoto, \& Suzuki 1995), and the companion star will spiral-in into the envelope of the Cas A progenitor. In order for most of the $\mathrm{H}$-rich envelope to be removed, the envelope should have been a red-giant size so that the orbital energy released during the spiral-in exceeds the binding energy of the envelope. After losing its envelope due to frictional heating, the star became a WN star.

If we take the model of $M_{\mathrm{MS}}=25 M_{\odot}$, as an example, the star at the WN stage has $8 M_{\odot}$. Since the explosion ejects $\mathrm{Si}$ and $\mathrm{Fe}$ from the deep layers (Hughes et al. 2000 ), the mass of the compact remnants could not exceed $2-3 M_{\odot}$. Then the binary system is very likely to be disrupted at the explosion. Then the compact star in the Cas A remnant does not have a companion star, and so no mass transfer can be postulated. The implication is also that the accretion onto the compact remnant can occur only as a result of fallback of the ejected matter, and so the composition of the fall back matter is mostly heavy elements with possibly a small fraction of helium but no hydrogen.

In either the single or binary scenario, the $\mathrm{WN}$ star blows a fast wind which collides with the red-giant wind material to form a dense shell (Chevalier \& Liang 1989). If the red-giant wind formed a ring-like shell (due possibly to the spiral-in of the companion), the collision between the supernova ejecta and the shell could explain the observed ring-like structure of Cas A.

\section{DISCUSSION AND CONCLUSION}


We agree with Chakrabarty et al. (2000) that for Cas A point source the usual ADAF model for a quiescent GBHC hardly reconcile with observation. However, we emphasized in $\S 2$ that there does exist a very promising $\mathrm{BH}$ disk accretion model. In this model, the fallback material is like the soft state of a GBHC with a disk-corona configuration, not like a quiescent GBHC with ADAF. With the low accretion rate and Comptonization of cooler soft photons $(\sim$ $0.1 \mathrm{keV}$ or less), we naturally obtain large photon index of $\Gamma \sim 2.6-4.1$ and lower luminosity of $L \sim 10^{34}-10^{35} \mathrm{erg}$ $\mathrm{s}^{-1}$, as observed from the Cas A point source.

Accreting NS models are also possible (see Chakrabarty et al. 2000). However, we can still, without difficulty, distinguish between the $\mathrm{BH}$ and NS accretion models because the characteristic properties of the observed X-ray spectra in these two cases are quite different (e.g., see Tanaka 2000). For instance, the radiation from an accreting NS is dominated by thermal emission from the stellar surface (Rutledge et al. 2000), which is absent if a BH is involved.

If the point source is a NS, the dominant radiation observed by Chandra most likely corresponds to the radiation from a localized small area. The detailed studies of theoretical light curves expected from anisotropic cooling of a NS have been carried out by, e.g., Shibanov et al. (1995) and Tsuruta (1998), with the latter including hot spots. The results show that pulsation depends on the relative angles between the rotation axis, magnetic axis and the line of sight. Depending on the combinations of these angles, pulsations from zero to up to about $30 \%$ are predicted, and so the observed constraints on the pulsed fraction are still consistent with a NS model.

Although the current data of Cas A point-source can be consistent with both $\mathrm{BH}$ and NS scenarios, future observations by the Chandra, XMM, and other satellite missions should be able to distinguish between these cases. If distinct periodicity is found the point source definitely should be a NS. The existence of the NS itself will significantly constrain the progenitor scenario for Cas A. Better spectral information should be able to distinguish between the $\mathrm{BH}$ and $\mathrm{NS}$ as the compact remnant. If the source is found to be a $\mathrm{BH}$, the implication is significant in the sense that this will offer the first observational evidence for $\mathrm{BH}$ formation through a SN explosion and greatly constrain the $\mathrm{BH}$ progenitor mass by combining with the abundance analysis of Cas A (Hughes et al. 2000).

In conclusion we emphasize that the Cas A point source can potentially provide great impacts on the theories of supernova explosion, progenitor scenario, compact remnant formation, accretion to compact objects, and NS thermal evolution.

We thank Drs. G. Pavlov, M. Rees, H. Tananbaum, B. Aschenbach, and J. Trümper for valuable discussions. This work has been supported in part by the grant-in-Aid for Scientific Research (0980203, 09640325), COE research (07CE2002) of the Ministry of Education, Science, Culture and Sports in Japan, and a NASA grant NAG5-3159.

\section{REFERENCES}

Aschenbach, B. 1999, IAUC No. 7249

Ashworth, W.B. 1980, J. Hist. Astron., 11, 1

Chakrabarty, D., Pivovaroff, M.J., Hernquist L E Heyl, J.S., \& Narayan, R. 2000, ApJ, submitted (astro-ph/0001026)

Chevalier, R.A., \& Liang, E. 1989, Ap J, 346, 847

Ergma, E., \& van den Heuvel, E.P.J. 1998, A\&A, 331, L29

Fesen, R.A., Becker, R.H., \& Blair, R.H. 1987, ApJ, 313, 378

Friedman, B., \& Pandharipande, V.R. 1981, Nucl. Phys. A, 361, 502

Holt, S., Gotthelf, E.V., Tsunemi, H., \& Negoro, H. 1994, PASJ, 46, L151

Holt $S$ et al 2000

http://asc.harvard.edu/targets/summary_observed_daily.htm

Hughes, J.P., Rakowski, C.E., Burrows, D.N., \& Stane, P.O. 2000, ApJ, 528, L109

Mineshige, S., Kusunose, M., \& Matsumoto, R.. 1995, ApJ, 445, L43

Mineshige, S., Nomoto, K., \& Shigeyama, T. 1993, A\&A, 267, 95

Mineshige, S., Nomura, H., Hirose, M., Nomoto, K., \& Suzuki, T. 1997, ApJ, 489, 22

Mineshige, S., \& Wheeler, J.C. 1989, ApJ, 343, 241

Narayan, R., McClintock, J.E., \& Yi, I. 1996, ApJ, 457, 821

Nomoto, K., Iwamoto, K., \& Suzuki, T. 1995, Phys. Rep., 256, 173

Nomoto, K., \& Tsuruta, S. 1987, ApJ, 312, 711
Pacini, F. 2000, in IAU Symp. 195, Highly Energetic Physical Processes and Mechanisms for Emission from Astrophysical Plasmas, eds. P. Martens, S. Tsuruta, \& M. Weber, PASP, in press

Pavlov, G.G., Zavlin, V.E., Aschenbach, B., \& Trümper, J..E. 2000, ApJ, 531, L53

Pringle, J.E. 1974, Ph.D. Thesis, University of Cambridge

Rutledge, R.E., Bildsten, L., Brown, E.F., Pavlov, G.G., \& Zavlin, V.E. 2000, ApJ, 529, 985

Schaller, G., Schaerer, D., Meynet, G., \& Maeder, A. 1992, A\&AS, 96,269

Shibanov, Yu.A., Zavlin, V.E., Pavlov, G., Qin, L., \& Tsuruta, S. 1995, Proc. 17th Texas Symp., eds. H. Böhringer, et al., N.Y. Acad. Sci., 291.

Tanaka, Y. 2000, in IAU Symp. 195, Highly Energetic Physical Processes and Mechanisms for Emission from Astrophysical Plasmas, eds. P. Martens, S. Tsuruta, \& M. Weber, PASP, in press

Tanaka, Y., \& Lewin, W. H. G., 1995, in X-ray binaries, ed. W.H.G. Lewin, J. van Paradijs, E.P.J. van den Heuvel (Cambridge: U.P., Cambridge), 126

Tananbaum, H. et al. 1999, IAUC No. 7246

Tsuruta, S. 1998, Phys.. Rep. 292, 1

Umeda, H., Nomoto, K., Tsuruta, S., Muto, T., \& Tatsumi, T. 1994, ApJ, 431, 309 (U94)

Umeda, H., Tsuruta, S., \& Nomoto, K. 1994, ApJ, 433, 256 (UTN94)

van den Bergh, S., \& Pritchet, C.J. 1986, ApJ, 307, 723 


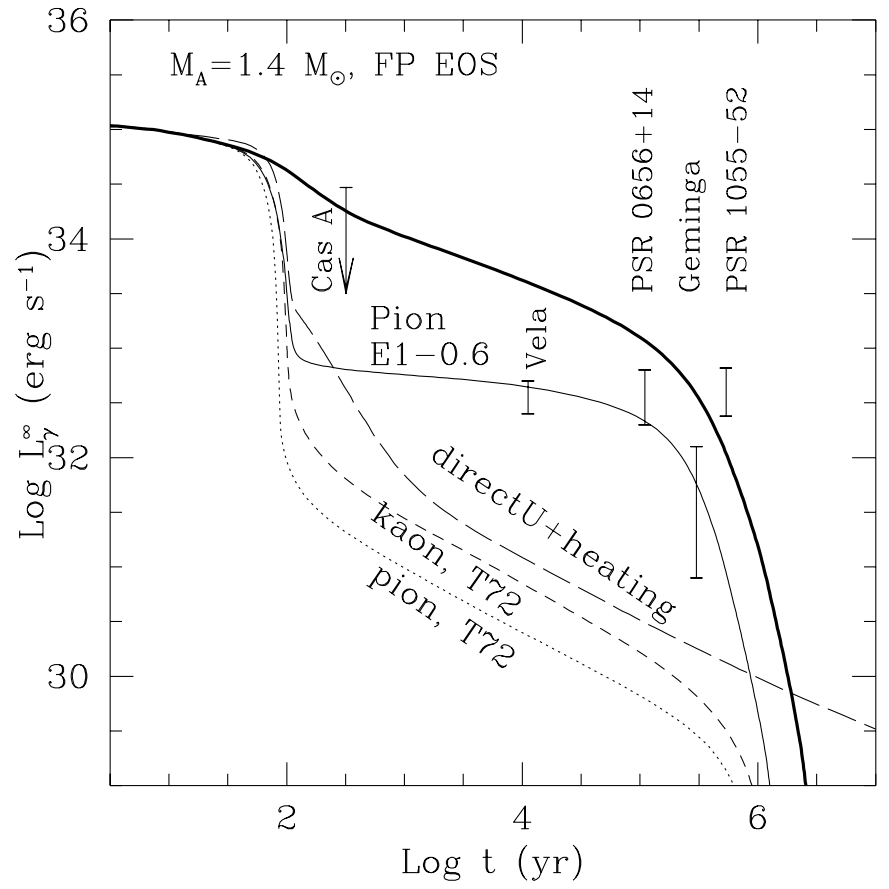

Fig. 1.- Various neutron star thermal evolution curves are compared with the observational data of the Cas A point source and several cooling NS candidates. The heavy solid curve refers to 'standard-cooling', the dashed and dotted curves show the 'non-standard' kaon and pion cooling scenarios when the superfluid effect is negligible, while the thin sold curve refers to the pion cooling with significant superfluid effect. The long dashed curve shows the effect of strong heating on the direct URCA cooling, the fastest 'non-standard' scenario. These curves are obtained for $1.4 M_{\odot}$ neutron stars with the intermediate FP equation of state (Friedman \& Pandharipande 1981). Cooling due to various more straightforward neutrino mechanisms such as the modified URCA is called 'standard cooling', while extremely fast cooling caused by some other more unconventional mechanisms is called 'non-standard cooling'. The Cas A data should be considered as the upper limit to the radiation from the whole stellar surface. 